\title{
Association between Hearing Loss and Vestibular Disorders: A Review of the Interference of Hearing in the Balance
}

\author{
Tatiana G. T. Santos ${ }^{1}$, Alessandra Ramos Venosa ${ }^{2}$, Andre Luiz Lopes Sampaio² \\ ${ }^{1}$ University of Catholic Medical School, Brasilia, Brazil \\ ${ }^{2}$ Department of Otolaryngology, University Hospital of Brasilia, Brasilia, Brazil \\ Email: tatiguthierre@hotmail.com
}

Received 20 March 2015; accepted 10 April 2015; published 17 April 2015

Copyright (C) 2015 by authors and Scientific Research Publishing Inc.

This work is licensed under the Creative Commons Attribution International License (CC BY). http://creativecommons.org/licenses/by/4.0/

(c) (i) Open Access

\begin{abstract}
Introduction: Dizziness is very prevalent and makes a great impact on people's life. Because of anatomical and functional similarities of hearing and vestibular systems, it is noted that there is a big relation between hearing loss and vestibular disorders. Depending on the age onset of hearing loss, it can cause even delay on motor development. Objective: To find literature that demonstrates the relation between hearing and balance. Confirming that hearing loss or even intervention to improve quality of hearing can interfere on vestibular system. Methodology: Revision of literature was carried out, preferring recent research only in English. Conclusion: Cochlea and vestibular systems have a close relationship; changes in one of them can cause big damage in the other. So, a complete evaluation of vestibular system is recommended before ear surgeries. Video Head Impulse Test is a new procedure able to evaluate high frequency movements of the head. It was an additional exam of vestibular status and came to help detect problems that were not diagnosed before. Efforts must be directed in order to protect the balance.
\end{abstract}

\section{Keywords}

Hearing Loss, Cochlear Implant, Vestibular Disorders, Dizziness and Vertigo

\section{Introduction}

Dizziness is among the most frequent symptoms in emergency room patients as demonstrated by Royl et al. in 2011 [1]. The management must be fast and effective due to similarities to central causes and to the great discomfort it causes to the patients. To make it possible, diagnostic tests must evolve. 
The American Speech-Language-Hearing Association reports that the percentage of newborns with permanent hearing loss at birth is about $3 \%$ and that the number increases to $6 \%$ by the time children get old [2]. Almost $70 \%$ of children presenting with sensorineural hearing loss (SNHL) have vestibular system disturb, with $20 \%-40 \%$ having severe bilateral vestibular loss. This population must be rehabilitated early in order to avoid motor and cognitional delayed development.

There are many causes of vestibular disorders. In this article, we will discuss about vestibular disorders related to: congenital hearing loss, age and surgical manipulation of the inner ear.

In adults, intervention must be quick to decrease long-term damages of auditory system and avoid interruption of their daily activities, increasing the patients' social interaction. The longer the time of hearing loss is, the higher the social impact of dizziness on the social life is. It can make patients change or quit work, reduce efficiency at work, disrupt in their social life, and suffer family difficulties, difficulties in travel, depression distress and anxiety, as reported by Beck in 2013 [3]. Seven years before Enticott had said that older patients seemed more susceptible to permanent injury after cochlear implantation surgery and it must be a great point of interest as the number of these patients submitted to that surgery was growing [4]. That group must be very well investigated previously the CI and followed after surgery as the electrical stimulation in that area can damage the vestibular system.

Although auditory and vestibular systems being distinct, they work just alike. So, there is a great relation among their functions. Once one is stimulated, the other suffers alteration as well.

\section{Methodology}

Revision of literature using Pubmed, Scopus and Web of Science and the keywords: hearing loss, cochlear implant, vestibular disorders, dizziness and vertigo. It was used only papers in English. It was selected 26 articles.

\section{Results}

There is a great association between vestibular and balance disorders with SNHL; however, most professionals do not routinely investigate for vestibular dysfunction among children with SNHL. The prevalence of vestibular and balance disorders in children is frequently underestimated, and it may range up to $15 \%$. Estimates indicate that almost $70 \%$ of children presenting with SNHL have vestibular system disturb, with $20 \%-40 \%$ having severe bilateral vestibular loss. This population will often have delayed motor development. They may evolve delayed development demonstrating poor head control beyond 6 weeks, delayed independent sitting beyond 9 months, and/or delayed walking beyond 18 months. If the clinician who examines those children inquires about motor milestones in case of presenting SNHL may do it correctly when, indeed, there is a true cause for concern, since SNHL relates to vestibular disturbs, that can cause motor delayed development [2].

Many identifiable etiologies of hearing loss have well-described associated vestibular impairments. They are in complete partition (type 1 to type 3), enlarged vestibular aqueduct syndrome, Usher Syndrome (the most important genetic diagnosis associated with vestibular impairment), meningitis, congenital CMV infection and children treated with ototoxic agents, such as aminoglycosides and chemotherapeutics. These children may present vestibular impairment progressive in nature, similar to the hearing loss. When progressive vestibular impairment occurs, these children may evolve with true vertigo, which can be severe and may last days or weeks. Vestibular impairment may occur associated with progression of hearing loss or independent of changes in hearing [2]. There is a suspicion that many of the children with cochlear nerve deficiency will also grow up with vestibular impairment on the ipsilateral side, and that is because of the relationship between auditory and vestibular systems [2]. Another study reveals that in the beginning of development, children depend on the visual system to maintain balance. Inoue et al. in 2013 evaluated that as they grow older, they progressively begin to use somatosensory and vestibular information until these systems reach full maturity around the age of 10 years. Children with profound hearing loss have dysfunction of the inferior as well as the superior vestibular nerve system and they show delayed acquisition of gross motor function [5].

In the same year, Cushing et al. said that children with SNHL may demonstrate associated vestibular impairment due to peripheral auditory and vestibular systems similarities. In a prospective and cross-sectional study were tested caloric, rotational stimuli responses of Horizontal canal and VEMP. One hundred fifty-three children with profound SNHL were tested; 119 had unilateral CI at time of testing, and 34 had no CI at the time of testing. Half of all children with profound SNHL presented vestibular end organ dysfunction. One third of the 
subjects displayed severe abnormalities. Vestibular end-organ dysfunction has great relation with etiology, meningitis and cochleovestibular anomalies have the highest rates of severe dysfunction. The ability to identify vestibular dysfunction in children with SNHL is very important because it allows prognostication of motor abilities and implementation of early therapy [6].

Ciuman made a review in 2011 describing that there are approximately 50 - 80 stereocillia and one kinocilium per vestibular hair cell. In contrast to vestibular system, the cochleacells does not have the kinocilium. The Glycocalyx and many others links keep all cells connected in both systems, despite of having different functions [7].

The auditory neuropathy is a disorder characterized by absent orseverely abnormal auditory brainstem responses withintact outer hair cell function, as seem in evoked otoacoustic emissions (OAE) and/or cochlearmicrophonics. Its etiology is multifactorial. The vestibular branch of the vestibulocochlear nerve, including the superior and the inferior vestibular nerves, is involved in maintaining a sense of equilibrium. The superior vestibular nerve and the semicircular canals can be assessed using electronystagmography (ENG): the inferior vestibular nerve and the saccule by the vestibular-evoked myogenic potentials. Sinha et al. two years later reviewed that the neuropathic condition in individuals with auditory neuropathy may additionally involve the vestibular nerve, and in most cases, the pathology is restricted to the peripheral vestibular system and does not affect the central oculomotor system [8].

Fujimoto et al. in 2014 published that approximately $40 \%$ of patients with idiopathic sudden hearing loss (ISHL) also suffer from vestibular symptoms which may occur at the onset of hearing loss or be delayed for hours or even days. Vertigo is more frequent in association with profound hearing loss and the recovery of hearing is worse in patients with vertigo than those without vertigo. The percentage of abnormal responses in patients with ISHL with vertigo was higher in the cVEMP test, followed by the oVEMP and caloric tests. These results suggest that the vestibular end organs closest to the cochlea tend to be preferentially affected in ISHL with vertigo [9].

Cochlear implant recipients usually complain of postoperative symptoms of dizziness. Although the auditory and vestibular systems are clearly distinct from one another, the mechanisms of neural transmission are identical. For this reason, the electrical stimulation through the cochlear implant may have an effect both on the auditory and vestibular systems. In a research it was described that $18 \%$ of 55 cochlear implant recipients saw connection between dizziness and the activation of the cochlear Implant. This gave grounds for supposing that the electricity spreads diffusely and could therefore stimulate the nerve endings of the vestibular nerve. Schwab et al. in 2010 found postoperatively that 33 patients $(66 \%)$ had no vestibular symptoms, whereas 17 (34\%) reported temporary symptoms of dizziness. Of these, eight patients $(16 \%)$ had showed corresponding symptoms prior to surgery. Only a small percentage complained of sensations as paresthesia and irritation of the facial nerve during the activation of the cochlear implant [10].

Although this surgery is considered to be safe, balance disorder is a very frequent postoperative complaint in cochlear recipients. In 2013, Katsiari et al found that about one-third of them could experience a significant vestibular disturbance after surgery, independently of age, cause, or preoperative caloric result. Even cochlear implantation leading to measurable changes of the peripheral vestibular function, permanent vertigo is rare. Different etiologies can cause vertigo after the surgery: perilymphatic fistula induced by cochlear fenestration or a disruption of endolymphatic flow caused by the electrode itself, mechanical irritation of the membranous labyrinth or thelabyrinthitis triggered by aforeign body in the cochlea. Histopathological studies on temporal bones implanted have shown that cochlear implantation can damage the vestibular end organ. In summary, it is clear that electrical stimulation affect vestibular system function [10] [11].

Hui-Chi Tien et al. in 2002 evaluated that the overall incidence of vestibular end-organ damage after cochlear implantation was $54.5 \%$, a higher estimative considering the literature based on history and/or functional tests. It must be considered the possibility of vestibular malfunction becaused by disease processes long before surgery. However, it can be consequence of trauma, associated infection, interruption of the endolymphatic system, hemorrhage, or vascular changes produced by insertion of implant electrodes. The saccule is the most frequent site of damage in the vestibular system, followed by the utricle and semicircular canals. It is safer to keep the electrode array in the scala tympani in order to minimize further vestibular damage. As the clinical incidence of balance disturbances after cochlear implantation is low, it demonstrates that damage of vestibular organs may occur and be asymptomatic [12].

During cochlear implant surgery planning, many items are considerate, one of which is the ear with poorer vestibular function. This requisite is important and normally selected as the site for implantation since surgery 
carries a low risk of iatrogenic labyrinthine injury. Hugh et al. in 2011 created an algorithm to help determine reasons to the choice of the better balance ear to implant. Reasons were divided into four categories: anatomical contraindications, attempting to attain binaural hearing, avoiding implantation of an ear with marked auditory deprivation, and patient preference. More studies are recommended and validation of that algorithm must be developed [13].

The study of incidence of vestibular dysfunction after cochlear implantation as well as its reasons is a big point of interest. It could help us to give better preoperative counseling and postoperative vestibular therapy to cochlear implant patients. Enticott et al. conducted a group in 2006 analyzing postoperative vestibular disturbance, defined as symptoms lasting for 1 week or longer after surgery [4]. The data in preoperative and postoperative vestibular function were measured from subjective assessments (Dizziness Handicap Inventory and Activity Balance Confidence questionnaires) and objective assessments (bithermal caloric tests). The information of the postoperative position of electrode was also classified as "loose", "regular", or "tight" fitting. About one third of patients reported vestibular disturbance, presenting poorer DHI, Activity Balance Confidence, and caloric results in the implanted ear after surgery. It seems that age, cause of hearing loss, and preoperative caloric result did not predict postoperative vestibular symptoms. Older patients had significantly poorer caloric results on the implanted side after surgery and that was not related to the intracochlear electrodes position or surgeons. They seem more susceptible to permanent injury after cochlear implantation (CI) surgery [7]. The number of adults who are potential candidates for cochlear implantation in the world is high and continue to increase. In research by Lin et al. in 2012 there is a negative association between the magnitude of the gain in speech scores and age at implantation, such that for every increasing year of age at CI the gain in speech scores was 1.3 percentage points less after adjusting for age at hearing loss onset. Individuals with higher pre-CI speech scores had significantly greater post-CI than those with lower pre-CI speech scores after adjusting for age at CI and age at hearing loss onset. In the future, research of CI in older patients should expand its evaluations to take into account the broad cognitive, social and physical outcomes that are likely detrimentally impacted by hearing loss [14].

Fina et al. in 2003 reported a group of 39\% (29/75) of subjects with implants that were dizzy after implantation. It was more common in older patients, individuals with previous dizziness history, and those older at onset of the hearing loss. The majority of subjects experienced dizziness in a delayed episodic fashion. Dizziness had no relation to implant activation. There are some subjects characteristics that have higher probability of experiencing disturbing vestibular symptoms after cochlear implantation as preimplantation vestibular symptoms, especially Ménière's Disease; age at implantation greater than 59 years old; age at onset of hearing loss greater than 26 years old and preimplantation abnormal computerized dynamic posturography [15]. Specific surgical complications reported in the literature include an electrode dislocation, facial nerve lesion, apart from inflammation and bleeding, but also dizziness and taste disorders. Wagner et al. in 2010 evaluated whether there is a higher incidence of vestibular and taste disorders after bilateral as compared to unilateral cochlear implantation. They found that specific testing showed in one case (5\%) a unilateral taste disorder after ipsilateral cochlear and that there is a higher risk for subjective vertigo after the second implantation. To increase patients' and medico legal safety in the procedure, the occurrence of unilateral and/or bilateral vestibular dysfunction and the potential risk of taste disorder should be included in the risk counseling before bilateral CI [16].

Other study by Steenerson et al. in 2001 evaluating vertigo after CI shows that imbalance was common preoperatively, positional vertigo was a common sequel postoperatively in the side of the implanted ear, responding well to vestibular therapy, and long-term intermittent vertigo or imbalance was rare in this group [17]. Vertigo and dizziness are among the risks after a CI surgery. It can occur soon after the surgery or after the implant activation. Bujang et al. in 2013 related that the postural stability of the experimental CI group have no significant difference with the normal control group in most tests situations, except in one specific test situation in which both the visual and somatosensory imputs was interrupted and modified. This study proposed that the exam that involves all the sensory measures is sensitive in evaluating the posture stability [18]. In 2001 Kubo et al. studied a group of 94 patients that presented the three types of dizziness after CI in 49\% of the individuals [19]. This incidence is considerably higher than some previously reported, but is similar to the value reported by Ito in 1998 [20], in which $47 \%$ reported some kind of subjective dizziness. Those three types of dizziness occur immediately or soon after the surgery but lasting for a few weeks, lasting several months due to bilateral deficits in vestibular function and vertigo of delayed onset.

In relation to pain and dizziness after pediatrics CI, Birman et al. in 2015 documented that children tolerate 
this surgery well. They experience little postoperative pain only requiring paracetamolfor few days after discharge from the hospital. The ones submitted to the bilateral procedure usually require it for a longer time, few more days, as well as the younger patitens. Slight dizziness was reported by $8 \%$ of all children at 1 week after surgery. No child had marked dizziness or unsteadiness. Four children had large vestibular aqueducts on radiology scans, two $(50 \%)$ of these children has slight unsteadiness at 1 week postoperatively [21].

Cochlear implantation has become more used recently to rehabilitate deaf born and postlingually deaf people. The acute, short-term dizziness after cochlear implantation seems to result primarily from a transient vestibular deficit of various origins. In contrast, chronic persisting dizziness after this surgery is largely based on a dysfunction of the saccular macula, which is an integral component of the otolith system. The data of Basta et al. in 2008 demonstrate that the saccular function can be profoundly influenced by cochlear implantation, and that surgical approaches trying to preserve saccular function are currently under development to prevent more damages. In the present original paper, they discuss also the possible coactivation of the inferior vestibular nerve by the electrical stimulation playing an additional role in the pathogenesis of the persisting postsurgical dizziness [22].

One year later Krause et al. evaluated patients with caloric and rotational chair vestibular function tests before and after CI surgery, which used the same approach. CI impairs the function of the horizontal semicircular canal (hSCC) but this alteration did not lead to vertigo complaints, so that no direct correlation could be established. Besides morphological changes, a CI also causes functional damage of vestibular parts of the labyrinth, but these findings did not lead to vertigo complaints. Other senses as visual afferents and central vestibular compensatory mechanisms play a role in order to the patient stay asymptomatic [23].

Vertigo and dizziness must always be a concern relating to complications after CI, even being usually a short-term symptom. With this in mind, Todt et al. in 2008 investigated the impact of different cochleostomy techniques on vestibular receptor integrity and vertigo after CI. They concluded that the round window approach for electrode insertion should be preferred to decrease the risk of vestibular dysfunction and vertigo [24].

In a study of Parmar et al. in 2012 they evaluated patients prior to CI surgery with preoperative bithermal caloric testing. One hundred seventy-seven patients were included, 148 in group A (CI in the ear with worse vestibular function) and 29 in group B (those implanted in the ear with better vestibular function). There was no significant difference in the frequency of dizziness before or after CI among groups, as well as the duration of dizziness postoperatively. This study has not searched for delayed onset dizziness, which has been documented in up to $40 \%$ of patients. The cause of such dizziness is often unknown, but up to $2 \%$ develop benign positional paroxysmal vertigo. As they used bithermal caloric testing, this means that the function of the utricle, saccule, and other semicircular canals was not tested. In addition, caloric testing may not entirely reflect the physiological function of the lateral canal as it mimics only low frequency stimulation [25].

Since cochlear surgical intervention is related to vestibular disturbs, it is extremely necessary doing the complete exam before intervention. As it works over a wide range of frequencies, it is important to test as many as possible. There is the caloric test, sensible to low frequencies $(0.004 \mathrm{~Hz})$ and the Rotary Chair Test to low to mid frequencies $(0.01-1.28 \mathrm{~Hz})$. The cVEMPs (cervical Vestibular evoked Myogenic Potentials) and oVEMPs (ocular Vestibular evoked Myogenic Potentials) have proven useful to evaluate the saccule and the inferior branch of the vestibular nerve, and the utricle and superior branch of the vestibular nerve, respectively. A new test is being implemented for high frequencies; the video head impulse test. This test relates to frequencies between 1 and $10 \mathrm{~Hz}$.

Jutila et al in the same year determine change in vestibular function in patients receiving a unilateral cochlear implant by Motorized Head Impulse Test (MHIT) and other signs and symptoms. Horizontal high-frequency vestibuloocular reflex (VOR) was measured preoperatively and twice postoperatively with no significantly change. Quality of life score did not change significantly also. Late high-frequency loss of vestibular function or vestibular symptoms is rare but possible after cochlear implantation surgery, and must be considered in counseling especially in bilateral CI surgery [26].

\section{Discussion}

Anatomical, histologic and physiologic similarities between the cochlear and vestibular end organs explain the relation between hearing loss and vestibular disturbs. As both systems are related, in patients with hearing loss it is prior to proceed a complete study of balance in order to diagnose and prevent a worse vestibular problem. 
In children with profound SNHL and consequent vestibular disorders may present delayed motor development.

As those systems are similar and anatomically just beside the vestibular end organs, close to the cochlea, they tend to be preferentially affected in ISHL with vertigo, as well as it happens with the electrical stimulation of vestibular system after cochlear implantation.

Cochlear implant surgery is becoming a popular procedure and it must be a concern the vestibular exam in advance in order to prevent equilibrium disorders after surgery. As vestibular system has a great frequency range, different kinds of exams must be applied to make a complete examination. Most of time, damages of vestibular organs may occur after surgery without symptoms and this must be understood.

Nowadays the number of adults and older patients being submitted to cochlear implant is growing, this way vestibular disturbs also increase, what must be related to surgical approach, previous disease and vestibular electrical stimulation for the cochlear implant.

Not all vestibular disorders have relationship with hearing loss, as we see in vestibular neuritis and vestibular migraine.

We started the analysis of the cochlear implant candidate patients' of HUB (Brasilia University Hospital).

All patients will answer dizziness handicap inventory (DHI) and perform vHIT before and after the surgery. All results will be analyzed and compared to literature. Until now, one patient had the complete evaluation and despite DHI became worse, vHIT continued normal. The second exam was performed 10 days after the cochlear implant.

In a previously analysis we can infer that some patients complain about dizziness after surgery. The fact of vHIT remained normal does not means that the vestibular system was not damaged. Other frequencies of movement that are not tested in vHIT could be injured, requiring a different kind of exam in order to evaluate other frequencies.

\section{Conclusions}

The inner ear contains organs responsible for hearing and balance. Cochlea and vestibular systems have a close relationship. So, disturb in one of them can cause a big damage in the other. Congenitally severe hearing loss can cause a great vestibular disturbance in children. Depending on the etiology of hearing loss, the vestibular disease can be extremely severe. Even the cochlear implant, because of either surgical approach or vestibular electrical stimulation by the implant, can cause vertigo after surgery.

Before a surgical manipulation in the inner ear, in order to promote better hearing, a complete exam of the vestibular system is required. Video Head Impulse Test is a new procedure able to evaluate high frequency movements of the head. That exam was an additional investigation of vestibular status, being able to detect problems that were not diagnosed before. Therefore, it came to improve evaluation of vestibular symptoms and investigation should be used regularly to prevent vertigo and other balance disorders after cochlear implant surgeries.

\section{References}

[1] Royl, G., Ploner, C.J. and Leithner, C. (2011) Dizziness in the Emergency Room: Diagnoses and Misdiagnoses. European Neurology, 66, 256-263. http://dx.doi.org/10.1159/000331046

[2] Beck, D.L., Petrak, M., Madell, J.R. and Cushing, S.L. (2015) Update 2015: Pediatric Vestibular, Balance, and Hearing Disorders. The Hearing Review, 22, 14.

[3] Yuri Agrawal, Y., Ward, B.K. and Minor, L.B. (2013) Vestibular Dysfunction: Prevalence, Impact and Need for Targeted Treatment. Journal of Vestibular Research, 23, 113-117.

[4] Enticott, J.C., Tari, S., Koh, S.M., Dowell, R.C. and O’Leary, S.J. (2006) Cochlear Implant and Vestibular Function. Otology \& Neurotology, 27, 824-830. http://dx.doi.org/10.1097/01.mao.0000227903.47483.a6

[5] Inoue, A., Iwasaki, S., Ushio, M., Chihara, Y., Fujimoto, C., Egami, N. and Yamasoba, T. (2013) Effect of Vestibular Dysfunction on the Development of Gross Motor Function in Children with Profound Hearing Loss. Audiology \& Neurotology, 18, 143-151. http://dx.doi.org/10.1159/000346344

[6] Cushing, S.L., Gordon, K.A., Rutka, J.A., James, A.L. and Papsin, B.C. (2013) Vestibular End-Organ Dysfunction in Children with Sensorineural Hearing Loss and Cochlear Implants: An Expanded Cohort and Etiologic Assessment. Otology \& Neurotology, 34, 422-428. 
[7] Ciuman, R.R. (2011) Auditory and Vestibular Hair Cell Stereocilia: Relationship between Functionality and Inner Ear Disease. The Journal of Laryngology \& Otology, 125, 991-1003. http://dx.doi.org/10.1017/S0022215111001459

[8] Sinha, S.K., Barman, A., Singh, N.K., Rajeshwari, G. and Sharanya, R. (2013) Vestibular Test Findings in Individuals with Auditory Neuropathy: Review. The Journal of Laryngology \& Otology, 127, 448-451. http://dx.doi.org/10.1017/S0022215113000406

[9] Fujimoto, C., Egami, N., Kinoshita, M., Sugasawa, K., Yamasoba, T. and Iwasaki, S. (2014) Involvement of Vestibular Organs in Idiopathic Sudden Hearing Loss with Vertigo: An Analysis Using oVEMP and cVEMP Testing. Clinical Neurophysiology, 126, 1388-2457.

[10] Schwab, B., Durisin, M. and Kontorinis, G. (2010) Investigation of Balance Function Using Dynamic Posturography under Electrical-Acoustic Stimulation in Cochlear Implant Recipients. International Journal of Otolaryngology, 2010, Article ID: 978594, 7 p.

[11] Katsiari, E., Balatsouras, D.G., Sengas, J., Riga, M., Korres, G.S. and Xenelis, J. (2013) Influence of Cochlear Implantation on the Vestibular Function. European Archives of Oto-Rhino-Laryngology, 270, 489-495. http://dx.doi.org/10.1007/s00405-012-1950-6

[12] Tien, H.C. and Linthicum Jr., F.H. (2002) Histopathologic Changes in the Vestibule after Cochlear Implantation. Otolaryngology-Head and Neck Surgery, 127, 260-264. http://dx.doi.org/10.1067/mhn.2002.128555

[13] Hugh, S.C., Shipp, D.B., Chen, J.M., Nedzelski, J.M. and Lin, V.Y.W. (2011) When Do We Choose the "Better Balance” Ear for Cochlear Implants? Cochlear Implants International, 12, 190-193. http://dx.doi.org/10.1179/1754762811Y.0000000006

[14] Lin, F.R., Chien, W.W., Li, L., Niparko, J.K. and Francis, H.W. (2012) Cochlear Implantation in Older Adults. Medicine (Baltimore), 91, 229-241. http://dx.doi.org/10.1097/MD.0b013e31826b145a

[15] Fina, M., Skinner, M., Goebel, J.A., Piccirillo, J.F. and Neely, J.G. (2003) Vestibular Dysfunction after Cochlear Implantation. Otology \& Neurotology, 24, 234-242. http://dx.doi.org/10.1097/00129492-200303000-00018

[16] Wagner, J.H., Basta, D., Wagner, F., Seidl, R.O., Ernst, A. and Todt, I. (2010) Vestibular and Taste Disorders after Bilateral Cochlear Implantation. European Archives of Oto-Rhino-Laryngology, 267, 1849-1854. http://dx.doi.org/10.1007/s00405-010-1320-1

[17] Steenerson, R.L., Cronin, G.W. and Gary, L.B. (2001) Vertigo after Cochlear Implantation. Otology \& Neurotology, 22, 842-843. http://dx.doi.org/10.1097/00129492-200111000-00021

[18] Bujang, R., Wahat, N.H.A. and Umat, C. (2013) Posture Stability in Adult Cochlear Implant Recipients. Journal of Medical Sciences, 13, 86-94.

[19] Kubo, T., Yamamoto, K., Iwaki, T., Doi, K. and Tamura, M. (2001) Different Forms of Dizziness Occurring after Cochlear Implant. European Archives of Oto-Rhino-Laryngology, 258, 9-12. http://dx.doi.org/10.1007/PL00007519

[20] Ito, J. (1998) Influence of the Multichannel Cochlear Implant on Vestibular Function. Otolaryngology-Head and Neck Surgery, 118, 900-902. http://dx.doi.org/10.1016/S0194-5998(98)70295-5

[21] Birman, C.S., Gibson, W.P.R. and Elliott, E.J. (2015) Pediatric Cochlear Implantation: Associated with Minimal Postoperative Pain and Dizziness. Otology \& Neurotology, 36, 220-222. http://dx.doi.org/10.1097/MAO.0000000000000569

[22] Basta, D., Todt, I., Goepel, F. and Ernst, A. (2008) Loss of Saccular Function after Cochlear Implantation: The Diagnostic Impact of Intracochlear Electrically Elicited Vestibular Evoked Myogenic Potentials. Audiology and Neurotology, 13, 187-192.

[23] Krause, E., Louza, J.P.R., Hempel, J.M., Wechtenbruch, J., Rader, T. and Gürkov, R. (2009) Effect of Cochlear Implantation on Horizontal Semicircular Canal Function. European Archives of Oto-Rhino-Laryngology, 266, 811-817. http://dx.doi.org/10.1007/s00405-008-0815-5

[24] Todt, I., Basta, D. and Ernst, A. (2008) Does the Surgical Approach in Cochlear Implantation Influence the Occurrence of Postoperative Vertigo? Otolaryngology-Head and Neck Surgery, 138, 8-12. http://dx.doi.org/10.1016/j.otohns.2007.09.003

[25] Parmar, A., Savage, J., Wilkinson, A., Hajioff, D., Nunez, D.A. and Robinson, P. (2012) The Role of Vestibular Caloric Tests in Cochlear Implantation. Otolaryngology-Head and Neck Surgery, 147, 127-131. http://dx.doi.org/10.1177/0194599812442059

[26] Jutila, T., Aalto, H. and Hirvonen, T.P. (2012) Cochlear Implantation Rarely Alters Horizontal Vestibulo-Ocular Reflex in Motorized Head Impulse Test. Otology \& Neurotology, 34, 48-52. http://dx.doi.org/10.1097/MAO.0b013e318277a430 